\title{
Social norms motivate COVID-19 preventive behaviors
}

\author{
Matthew H. Goldberg, ${ }^{1, *}$, Abel Gustafson ${ }^{1}$, Edward W. Maibach², Sander van der Linden ${ }^{3}$, \\ Matthew T. Ballew ${ }^{1}$, Parrish Bergquist ${ }^{1}$, John E. Kotcher ${ }^{2}$, Jennifer R. Marlon ${ }^{1}$, \\ Seth A. Rosenthal ${ }^{1}$, and Anthony Leiserowitz ${ }^{1}$ \\ ${ }^{1}$ Yale Program on Climate Change Communication, Yale University \\ ${ }^{2}$ Mason Center for Climate Change Communication, George Mason University \\ ${ }^{3}$ Department of Psychology, Cambridge University
}

**This is a working paper and has not yet been peer-reviewed**

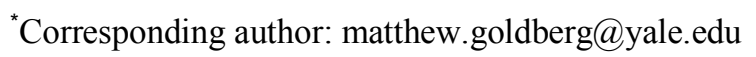




\section{Social norms motivate COVID-19 preventive behaviors}

Coronavirus disease 2019 (COVID-19) is a major global public health threat. Until a vaccination is available to biologically protect people from contracting and spreading the virus, social solutions are paramount. ${ }^{1}$ Social distancing and other preventive behaviors are projected to substantially reduce the number of COVID-19 cases and save many lives. ${ }^{2}$ Because there is substantial variability in people's adoption of the preventive behaviors recommended by experts and public health organizations, it is vital to understand the factors that motivate or inhibit adoption.

The conventional approach to promoting preventive behaviors in a public health crisis is “top-down" communication from authority figures, opinion leaders, and mainstream media outlets. However, some traditional, official information sources are perceived as partisan. This means that for some audiences, critical information about recommended behaviors comes from sources they do not trust.

Another strategy, although less frequently considered, is "horizontal" communication through personal relationships, social networks, and communities. This type of communication can affect behavior, in part through social norms including perceptions of whether (and how much) other people are engaging in preventive behavior, and whether other people think those preventive behaviors are important. This "horizontal" flow of information about preventive behaviors can be particularly effective because it comes from familiar sources that people trust. Social norms positively influence behavior in many other health contexts, such as diet, exercise, and smoking cessation. ${ }^{3}$ 
Here, we estimate the hypothesized causal effect of perceived social norms on individuals' COVID-19 preventive behaviors. To estimate the effect of increased social norms on preventive behaviors, we conducted a large national survey $(N=3,933)$ with quotas used to match census parameters on sex, race, age, education, income, and geographic region. Sampling weights were used to correct for small deviations from census parameters. We measured perceived social norms (i.e., how often family and friends perform preventive behaviors, and whether they think it is important for the respondent to do so), participation in preventive behaviors, and diverse variables that help control for potential confounds.

Estimating hypothesized causal effects with minimal bias using observational studies depends on knowledge of the common causes of the treatment and outcome in order to rule out alternative explanations. ${ }^{4,5}$ Much knowledge about social norms has been produced over the past few decades, enabling us to propose and measure a rich set of variables that represent the likely common causes of both social norms and COVID-19 preventive behavior. By controlling for these alternative explanations, we can estimate the hypothesized causal effect of social norms on preventive behaviors.

We used extant theory as well as COVID-19 behavioral science research to choose a list of potential common causes to control for in our analyses. We controlled for (a) sociodemographics (age, education, race/ethnicity); (b) location (geographic region; whether the respondent lives in a rural, suburban, or urban area) (c) political party and political ideology; (d) individualistic and egalitarian values; (e) media attention and exposure (how closely respondents say they are following news about COVID-19; how often they consume information from Facebook, Twitter, and YouTube); (f) health indicators (subjective personal health; respondents' beliefs about whether they have been in contact with someone who has COVID-19); (g) risk 
perceptions (timing of harm to their local community); (h) other social factors (frequency of discussion about COVID-19 with friends and family; perceived disease-preventive descriptive norms in one's local community,); (i) whether one lives with one or more people over 65 years old; and (j) the date and time the respondent completed the survey.

We used structural equation modeling to predict preventive behaviors, using the average of two items that measure perceived social norms among friends and family, while controlling for the set of potential confounders listed above. The hypothesized effect of social norms was substantial for most behaviors. For example, each unit increase in perceived social norms among friends and family predicted a doubling of the odds of performing each of eleven different preventive behaviors, including more frequently washing hands with soap and water, avoiding parties and other personal events, and staying at least six feet away from others when in public. Additionally, each unit increase in norms is expected to lead to $66 \%$ greater odds of wearing a mask when in public.

Figure 1. The predicted effect of social norms on preventive behaviors.

Preventive Behaviors Effect of Perceived Social Norms

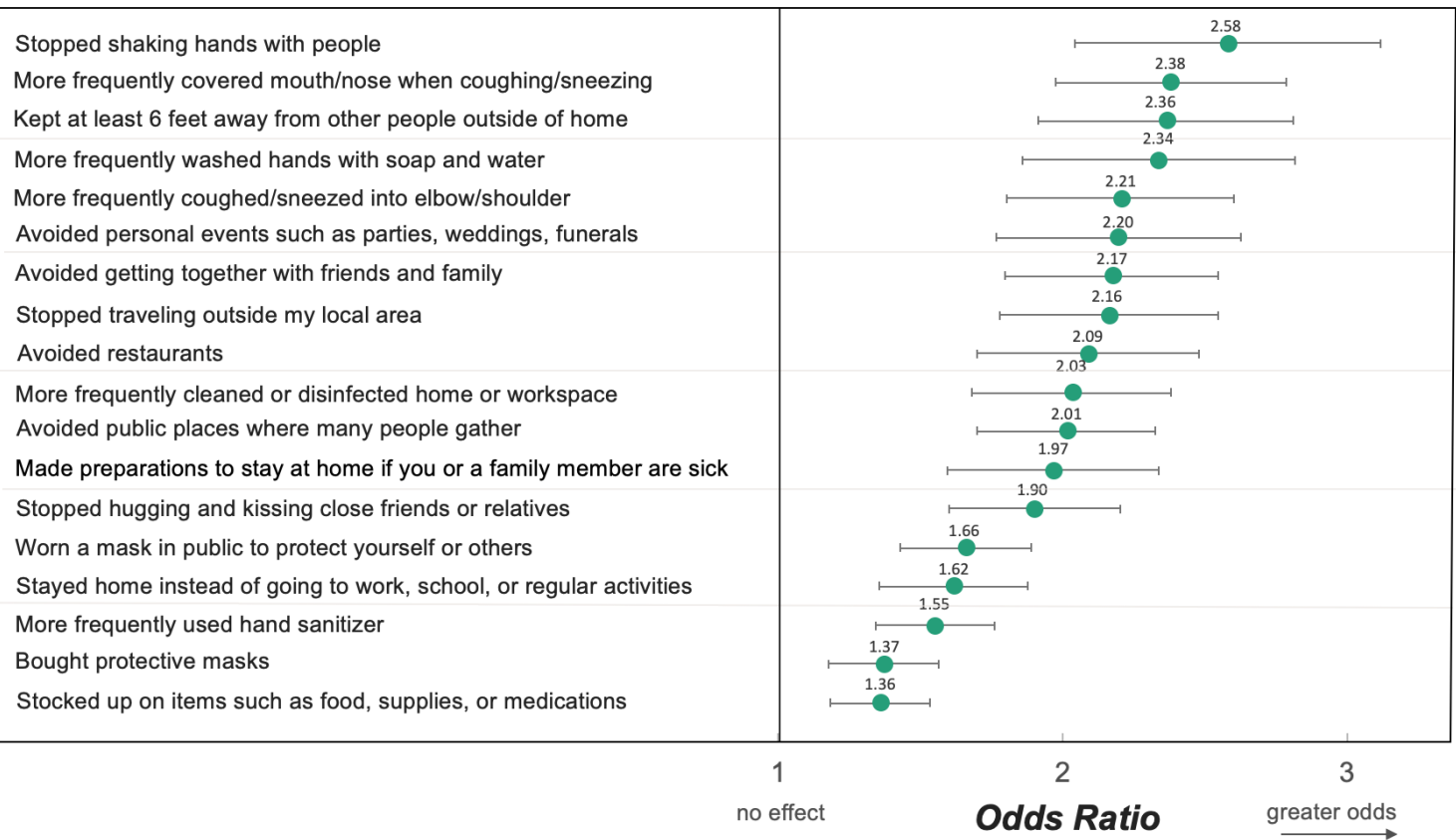


Note. Effects sizes are those observed while controlling for the set of covariates described in the main text. Error bars represent $95 \%$ confidence intervals. We report the results of 18 of the 23 preventive behaviors measured. The remaining 5 were excluded from this figure to preserve legibility and because many respondents indicated that that behavior "Does not apply" (e.g., "Chosen to keep children at home or away from others").

These findings highlight the crucial influence of social norms, specifically among friends and family. Importantly, increasing people's perceived norms is likely to lead them to adopt more preventive behaviors, and to perform them more frequently. For many preventive behaviors, we estimated that increases would be quite substantial, often doubling the odds that people will adopt the behavior.

Increasing the strength of norms can be done by increasing people's perceptions of the frequency of preventive behaviors that close social network members are doing (descriptive norms) as well as what close social network members approve of (injunctive norms). Both types of norms can be emphasized in communication among friends and family members. That is, the current findings suggest that the proportion of Americans performing preventive behaviors will substantially increase if people communicate to friends and family that they are engaging in these behaviors themselves, and that you should too, to keep yourself and others safe.

\section{References}

1. Fauci, A. S., Lane, H. C., \& Redfield, R. R. (2020). Covid-19—navigating the uncharted. New England Journal of Medicine, 1268-1269.

2. Anderson, R. M., Heesterbeek, H., Klinkenberg, D., \& Hollingsworth, T. D. (2020). How will country-based mitigation measures influence the course of the COVID-19 epidemic?. The Lancet, 395(10228), 931-934.

3. Sheeran, P., Maki, A., Montanaro, E., Avishai-Yitshak, A., Bryan, A., Klein, W. M., ... \& Rothman, A. J. (2016). The impact of changing attitudes, norms, and self-efficacy on 


\section{SOCIAL NORMS AND COVID-19 PREVENTIVE BEHAVIOR}

health-related intentions and behavior: a meta-analysis. Health Psychology, 35(11), 11781188.

4. Cook, T. D., Shadish, W. R., \& Wong, V. C. (2008). Three conditions under which experiments and observational studies produce comparable causal estimates: New findings from within-study comparisons. Journal of Policy Analysis and Management: The Journal of the Association for Public Policy Analysis and Management, 27(4), 724750.

5. Shadish, W. R., Clark, M. H., \& Steiner, P. M. (2008). Can nonrandomized experiments yield accurate answers? A randomized experiment comparing random and nonrandom assignments. Journal of the American Statistical Association, 103(484), 1334-1344. 